\title{
Synthesis and Characterisation of Two Different Series of Isomers of Mixed Ligand Alumininm Complexes Derived from Sterically Hindered Schiff Bases and Glycols.
}

\author{
Simpal Sharma, A.K. Rai and Yashpal Singh* \\ Department of Chemistry, University of Rajasthan, Jaipur-302004 India
}

\begin{abstract}
Two different series of isomers of new mixed ligand aluminium complexes containing a glycol moiety and bifunctional tridentate Schiff bases have been synthesized by different routes. Equimolar reactions of $\mathrm{Al}\left(\mathrm{OPr}^{\mathrm{i}}\right)_{3}$, glycol and substituted benzothiazoline give new mixed ligand glycolate derivatives of aluminium [OGOAl $\left.(\mathrm{OH}) \mathrm{C}_{6} \mathrm{H}_{3} \mathrm{XC}(\mathrm{R})=\mathrm{NC}_{6} \mathrm{H}_{4} \mathrm{~S}\right]$. But reactions of $(\mathrm{L}) \mathrm{Al}(\mathrm{OPr})$ with $\mathrm{G}(\mathrm{OH})_{2}$ yield different complexes, [ HOGOAIOC $_{6} \mathrm{H}_{3} \mathrm{XC}(\mathrm{R})=\mathrm{NC}_{6} \mathrm{H}_{4} \mathrm{~S}$ ], where $\mathrm{G}=\mathrm{G}^{\prime}=\left(-\mathrm{CMe}_{2} \mathrm{CH}_{2} \mathrm{CHMe}-\right)$ or $\mathrm{G}^{2}=\left(-\mathrm{CMe}_{2} \mathrm{CMe}_{2}-\right) ; \mathrm{R}=\mathrm{X}=\mathrm{H}$ $\left(\mathrm{L}^{\mathrm{i}} \mathrm{H}_{2}\right) ; \mathrm{R}=\mathrm{CH}_{3}, \mathrm{X}=\mathrm{H}\left(\mathrm{L}^{2} \mathrm{H}_{2}\right) ; \mathrm{R}=\mathrm{H}, \mathrm{X}=\mathrm{OCH}_{3}\left(\mathrm{~L}^{3} \mathrm{H}_{2}\right)$. All these derivatives have been characterized by elemental analyses, molecular weight measurements and their probable structures have been proposed on the basis of IR and NMR $\left({ }^{1} \mathrm{H},{ }^{13} \mathrm{C}\right.$ and $\left.{ }^{27} \mathrm{AI}\right)$ spectral studies.
\end{abstract}

\section{INTRODUCTION}

A number of mixed ligand aluminium compounds $/ 1-12 /$ have been reported in the literature. In most of these compounds, aluminium prefers to attain a co-ordination number four or six. However, when sterically hindered ligands are used, compounds with penta-coordinated aluminium are obtained $113-15 /$. In these derivatives, mostly monofunctional bidentate ligands have been used. Mixed ligand aluminium derivatives having a bifunctional tridentate ligand and a glycol moiety appear to be scanty. In view of the above, we have synthesized two different series of isomers of mixed ligand aluminium compounds based on bifunctional tridentate ligands, in which the aluminium atom is found to be penta-coordinated.

\section{MATERIALS AND METHODS}

All the chemicals used were of reagent grades. Moisture is carefully excluded throughout the experimental manipulations. $\mathrm{Al}\left(\mathrm{OPr}^{\prime}\right)_{s} / / 6 /$ and $(\mathrm{OGO}) \mathrm{Al}\left(\mathrm{OPr}^{\prime}\right) / 17 /$ were prepared by literature methods. 
Isopropyl alcohol in the azeotrope was determined oxidimetrically /18/, using $1 \mathrm{~N} \mathrm{~K}_{2} \mathrm{Cr}_{2} \mathrm{O}_{7}$ solution in $12.5 \%$. $\mathrm{H}_{2} \mathrm{SO}_{4}$. Aluminium was estimated as oxinate gravimetrically /18/, nitrogen and sulphur were determined by the Kjeldahl and Messenger methods respectively /18/. Glycols, 2-aminothiophenol, salicylaldehyde, 2hydroxy acetophenone and o-vanilline were distilled prior to use. Solvents (E. Merck) were purified and dried by standard procedure /19/. Benzothiazolines /20/ $\mathrm{HOC}_{6} \mathrm{H}_{3} \mathrm{XC}(\mathrm{R})-\mathrm{N}(\mathrm{H}) \mathrm{C}_{6} \mathrm{H}_{4} \mathrm{~S}\left(\mathrm{LH}_{2}\right)$ (where $\mathrm{LH}_{2}=$ $\mathrm{L}^{1} \mathrm{H}_{2}(\mathrm{R}=\mathrm{X}=\mathrm{H}) ; \mathrm{L}^{2} \mathrm{H}_{2}\left(\mathrm{R}=\mathrm{CH}_{3}, \mathrm{X}=\mathrm{H}\right) ; \mathrm{L}^{3} \mathrm{H}_{2}\left(\mathrm{R}=\mathrm{H}, \mathrm{X}=\mathrm{OCH}_{3}\right)$ ) were prepared by the equimolar condensation reaction of 2-aminothiophenol and substituted salicylaldehyde. ${ }^{1} \mathrm{H}(89.55 \mathrm{MHz}),{ }^{27} \mathrm{Al}(23.29$ $\mathrm{MHz})$ and ${ }^{13} \mathrm{C}(22.49 \mathrm{MHz}) \mathrm{NMR}$ spectra in $\mathrm{CDCl}_{3}$ solution were recorded on a JEOL FX90Q Spectrometer. IR spectra of these complexes have been recorded on CsI cell on a Nicolet DXFTIR Spectrophotometer. Molecular weights of soluble compounds were determined ebullioscopically in benzene solution using a Gallenkamp ebulliometer.

\section{Preparation of (OGOH)Al(L) and (OGO)AI(LH)}

As the synthetic procedure for these mixed ligand aluminium complexes is the same, the preparative details are being given for only one complex (le) of each series. Analytical details for analogous compounds are summarized in Table-I.

\section{(a) Preparation of $\left(O G^{2} O H\right) A I\left(L^{2}\right)$}

The compound $\left(\mathrm{OG}^{2} \mathrm{OH}\right) \mathrm{Al}\left(\mathrm{L}^{2}\right)$ was prepared in the following two steps:

\section{Preparation of $\left(\mathrm{L}^{2}\right) \mathrm{Al}\left(\mathrm{OPr}^{\mathrm{i}}\right)$}

The reaction mixture containing equimolar amounts of $\mathrm{Al}\left(\mathrm{OPr}^{i}\right)_{3}$ and $\mathrm{L}^{2} \mathrm{H}_{2}$ in benzene $(\sim 45 \mathrm{ml})$ was refluxed on a fractionating column for $\sim 24 \mathrm{~h}$, during which time the liberated isopropyl alcohol was continuously fractionated out azeotropically and determined (by the oxidimetric method /18/) periodically to monitor the progress and the completion of the reaction. When the azeotrope showed negligible presence of isopropyl alcohol, the reaction was stopped and the excess of solvent was removed under reduced pressure to afford a yellow solid which was purified by crystallization from a mixture of $\mathrm{CHCl}_{3}$ and petroleum ether $\left(40-60^{\circ} \mathrm{C}\right)$. The product on drying and analysis was found to have the composition $\left(\mathrm{L}^{2}\right) \mathrm{Al}\left(\mathrm{OPr}^{i}\right)$ in $89 \%$ yield.

\section{Preparation of $\left(\mathrm{HOG}^{2} \mathrm{O}\right) \mathrm{Al}\left(\mathrm{L}^{2}\right)$}

$\mathrm{G}^{2}(\mathrm{OH})_{2}(0.37 \mathrm{~g}, 3.16 \mathrm{mmol})$ was added to the benzene solution $(\sim 40 \mathrm{ml})$ of $\left(\mathrm{L}^{2}\right) \mathrm{AI}\left(\mathrm{OPr}^{\mathrm{i}}\right)(1.03 \mathrm{~g}, 3.16$ $\mathrm{mmol}$ ) and was refluxed on a fractionating column for $\sim 4 \mathrm{~h}$. The isopropanol formed in the reaction was fractionated out azeotropically with benzene. Progress as well as completion of the reaction was checked by the estimation of the isopropyl alcohol in the azectrope by the oxidimetric method /18/. After stripping off the solvent under reduced pressure, a yellow solid was obtained which was purified by recrystallization from a mixture of $\mathrm{CHCl}_{3}$ and petroleum ether $\left(40-60^{\circ} \mathrm{C}\right)$. Details of analytical data of analogous derivatives have been summarized in Table-I. Adopting the procedure similar to (le), other derivatives were prepared from ( $\mathrm{L}) \mathrm{Al}\left(\mathrm{OPr}^{\prime}\right)$ and appropriate $\mathrm{G}(\mathrm{OH})_{2}$. The amounts of the reactants $(\mathrm{g}$, mmol) used are shown in brackets: 
(la) : $\quad\left(\mathrm{L}^{\mathrm{l}}\right) \mathrm{Al}\left(\mathrm{OPr}^{\mathrm{i}}\right)(1.14,3.64)$ and $\mathrm{G}^{\prime}(\mathrm{OH})_{2}(0.43,3.64)$

(lb) : $\quad\left(\mathrm{L}^{2}\right) \mathrm{Al}\left(\mathrm{OPr}^{\mathrm{i}}\right)(1.11,3.39)$ and $\mathrm{G}^{1}(\mathrm{OH})_{2}(0.40,3.39)$

(1c): $\quad\left(\mathrm{L}^{3}\right) \mathrm{Al}\left(\mathrm{OPr}^{\mathrm{i}}\right)(1.09,3.20)$ and $\mathrm{G}^{\prime}(\mathrm{OH})_{2}(0.38,3.20)$

(Id) : $\quad\left(\mathrm{L}^{1}\right) \mathrm{Al}\left(\mathrm{OPr}^{\mathrm{i}}\right)(0.93,2.96)$ and $\mathrm{G}^{2}(\mathrm{OH})_{2}(0.35,2.96)$

(1f) : $\quad\left(\mathrm{L}^{3}\right) \mathrm{Al}\left(\mathrm{OPr}^{\mathrm{i}}\right)(1.02,2.96)$ and $\mathrm{G}^{2}(\mathrm{OH})_{2}(0.35,2.96)$

Table 1

Analytical data of mixed ligand aluminium complexes.

\begin{tabular}{|c|c|c|c|c|c|c|}
\hline \multirow{2}{*}{$\begin{array}{c}\text { Empirical formula of } \\
\text { complexes, colour, state } \\
\text { and yield }(\%) \\
\end{array}$} & \multirow{2}{*}{$\begin{array}{l}\text { PríH }(g) \\
\text { Found } \\
\text { (Calc.) } \\
\end{array}$} & \multicolumn{3}{|c|}{$\%$ Analysis Found (Calc.) } & \multirow[t]{2}{*}{ M.P. $\left({ }^{\circ} \mathrm{C}\right)$} & \multirow{2}{*}{$\begin{array}{l}\text { M.Wt. } \\
\text { Found } \\
\text { (Calc.) }\end{array}$} \\
\hline & & Al & $\mathbf{S}$ & $\mathbf{N}$ & & \\
\hline $\begin{array}{c}\mathrm{C}_{19} \mathrm{H}_{22} \mathrm{NSO}_{3} \mathrm{Al} \text { (Ia) Pale } \\
\text { yellow solid, } 90\end{array}$ & $\begin{array}{r}0.20 \\
(0.22) \\
\end{array}$ & $\begin{array}{r}7.20 \\
(7.26) \\
\end{array}$ & $\begin{array}{c}8.61 \\
(8.63)\end{array}$ & $\begin{array}{c}3.69 \\
(3.77)\end{array}$ & $132-140$ & - \\
\hline $\begin{array}{l}\mathrm{C}_{20} \mathrm{H}_{24} \mathrm{NSO}_{3} \mathrm{Al}(\mathbf{I b}) \\
\text { Deep yellow solid, } 91\end{array}$ & $\begin{array}{c}0.19 \\
(0.20)\end{array}$ & $\begin{array}{c}6.86 \\
(6.99) \\
\end{array}$ & $\begin{array}{r}8.30 \\
(8.31)\end{array}$ & $\begin{array}{c}3.52 \\
(3.63)\end{array}$ & $153-160$ & $\begin{array}{c}380 \\
(385.5)\end{array}$ \\
\hline $\begin{array}{c}\mathrm{C}_{20} \mathrm{H}_{24} \mathrm{NSO}_{4} \mathrm{AI} \text { (Ic) } \\
\text { Greenish yellow solid, } 90\end{array}$ & $\begin{array}{r}0.17 \\
(0.19) \\
\end{array}$ & $\begin{array}{r}6.69 \\
(6.72) \\
\end{array}$ & $\begin{array}{r}7.26 \\
(7.99) \\
\end{array}$ & $\begin{array}{r}3.38 \\
(3.49) \\
\end{array}$ & $\begin{array}{c}210-220 \\
\text { (Dec.) }\end{array}$ & - \\
\hline $\begin{array}{c}\mathrm{C}_{19} \mathrm{H}_{22} \mathrm{NSO}_{3} \mathrm{Al}(\mathbf{1 d}) \quad \text { Pale } \\
\text { yellow solid, 91 }\end{array}$ & $\begin{array}{c}0.16 \\
(0.18) \\
\end{array}$ & $\begin{array}{r}7.12 \\
(7.26) \\
\end{array}$ & $\begin{array}{r}8.56 \\
(8.63) \\
\end{array}$ & $\begin{array}{c}3.59 \\
(3.77) \\
\end{array}$ & $135-140$ & - \\
\hline $\begin{array}{c}\mathrm{C}_{20} \mathrm{H}_{24} \mathrm{NSO}_{3} \mathrm{Al}(\mathrm{le}) \\
\text { Yellow solid, } 90\end{array}$ & $\begin{array}{r}0.16 \\
(0.18) \\
\end{array}$ & $\begin{array}{r}6.82 \\
(6.99) \\
\end{array}$ & $\begin{array}{r}8.29 \\
(8.32) \\
\end{array}$ & $\begin{array}{r}3.52 \\
(3.63) \\
\end{array}$ & $155=160$ & $\begin{array}{r}381 \\
(385.5) \\
\end{array}$ \\
\hline $\begin{array}{c}\mathrm{C}_{20} \mathrm{H}_{24} \mathrm{NSO}_{4} \mathrm{AI}(\mathbf{I f}) \\
\text { Yellow solid, } 90 \\
\end{array}$ & $\begin{array}{r}0.16 \\
(0.17) \\
\end{array}$ & $\begin{array}{r}6.29 \\
(6.72) \\
\end{array}$ & $\begin{array}{r}7.56 \\
(7.99) \\
\end{array}$ & $\begin{array}{c}3.39 \\
(3.49)\end{array}$ & $\begin{array}{c}215-220 \\
\text { (Dec.) }\end{array}$ & - \\
\hline $\begin{array}{c}\mathrm{C}_{19} \mathrm{H}_{22} \mathrm{NSO}_{3} \mathrm{Al}(2 \mathrm{a}) \quad \text { Pale } \\
\text { yellow solid, } 94\end{array}$ & $\begin{array}{r}1.11 \\
(1.13) \\
\end{array}$ & $\begin{array}{r}7.24 \\
(7.26) \\
\end{array}$ & $\begin{array}{r}8.62 \\
(8.63) \\
\end{array}$ & $\begin{array}{c}3.65 \\
(3.77) \\
\end{array}$ & $130-135$ & - \\
\hline $\begin{array}{c}\mathrm{C}_{20} \mathrm{H}_{24} \mathrm{NSO}_{3} \mathrm{Al}(\mathbf{2 b}) \\
\text { Deep yellow solid, } 92\end{array}$ & $\begin{array}{r}1.17 \\
(1.27) \\
\end{array}$ & $\begin{array}{r}6.19 \\
(6.99) \\
\end{array}$ & $\begin{array}{r}8.20 \\
(8.31) \\
\end{array}$ & $\begin{array}{c}3.45 \\
(3.63) \\
\end{array}$ & $158-162$ & $\begin{array}{c}382 \\
(385.5) \\
\end{array}$ \\
\hline $\begin{array}{c}\mathrm{C}_{20} \mathrm{H}_{24} \mathrm{NSO}_{4} \mathrm{Al}(2 \mathrm{c}) \\
\text { Greenish yellow solid, } 92\end{array}$ & $\begin{array}{r}0.81 \\
(0.84) \\
\end{array}$ & $\begin{array}{r}6.70 \\
(6.72) \\
\end{array}$ & $\begin{array}{r}7.87 \\
(7.99) \\
\end{array}$ & $\begin{array}{r}3.44 \\
(3.49) \\
\end{array}$ & $\begin{array}{c}218-225 \\
\text { (Dec.) }\end{array}$ & - \\
\hline $\begin{array}{c}\mathrm{C}_{19} \mathrm{H}_{22} \mathrm{NSO}_{3} \mathrm{AI}(2 \mathrm{~d}) \quad \text { Pale } \\
\text { yellow solid, 91 }\end{array}$ & $\begin{array}{r}0.70 \\
(0.72) \\
\end{array}$ & $\begin{array}{r}7.21 \\
(7.26) \\
\end{array}$ & $\begin{array}{r}8.59 \\
(8.63) \\
\end{array}$ & $\begin{array}{c}3.72 \\
(3.77) \\
\end{array}$ & $135-140$ & - \\
\hline $\begin{array}{c}\mathrm{C}_{20} \mathrm{H}_{24} \mathrm{NSO}_{3} \mathrm{Al}(2 \mathrm{e}) \\
\text { Yellow solid, } 89 \\
\end{array}$ & $\begin{array}{c}1.13 \\
(1.14) \\
\end{array}$ & $\begin{array}{r}6.59 \\
(6.99) \\
\end{array}$ & $\begin{array}{r}8.21 \\
(8.31) \\
\end{array}$ & $\begin{array}{r}3.52 \\
(3.63)\end{array}$ & $152-155$ & $\begin{array}{c}381 \\
(385.5) \\
\end{array}$ \\
\hline $\begin{array}{c}\mathrm{C}_{20} \mathrm{H}_{24} \mathrm{NSO}_{4} \mathrm{Al}(2 \mathrm{f}) \\
\text { Yellow solid, } 90 \\
\end{array}$ & $\begin{array}{r}0.72 \\
(0.75) \\
\end{array}$ & $\begin{array}{r}6.49 \\
(6.72) \\
\end{array}$ & $\begin{array}{r}7.87 \\
(7.99) \\
\end{array}$ & $\begin{array}{c}3.47 \\
(3.49)\end{array}$ & $\begin{array}{c}215-220 \\
\text { (Dec.) }\end{array}$ & - \\
\hline
\end{tabular}

\section{(b) Preparation of $\left(O G^{2} O\right) A l\left(L^{2} H\right)$}

These compounds may be prepared by two different methods; since the synthetic procedure has been adopted for all the derivatives, one representative preparation (2e) is being given for the sake of brevity. 
(i) The reaction mixture containing equimolar amounts of $\left(\mathrm{OG}^{2} \mathrm{O}\right) \mathrm{Al}\left(\mathrm{OPI}^{\prime}\right)(1.23 \mathrm{~g}, 6.082 \mathrm{mmol})$ and $\mathrm{L}^{2} \mathrm{H}_{2}(1.47 \mathrm{~g}, 6.082 \mathrm{mmol})$ in benzene $(\sim 45 \mathrm{ml})$ was refluxed on a fractionating column for $\sim 24 \mathrm{~h}$. The isopropanol liberated during the course of the reaction was fractionated off azeotropically and determined (by the oxidimetric method /18/) periodically to monitor the progress and completion of the reaction. On completion, the reaction was stopped and excess of the solvent was removed under reduced pressure, yielding a yellow solid which was recrystallized from mixture of $\mathrm{CHCl}_{3}$ and petroleum ether $\left(40-60^{\circ} \mathrm{C}\right)$ at $-20^{\circ} \mathrm{C}$ in $88 \%$ yield.

(ii) The reaction mixture containing equimolar amounts of $\mathrm{Al}\left(\mathrm{OPr}^{\prime}\right)_{3}(1.29 \mathrm{~g}, 6.31 \mathrm{mmol}), \mathrm{G}^{2}(\mathrm{OH})_{2}(0.75$ g, $6.31 \mathrm{mmol})$ and $\mathrm{L}^{2} \mathrm{H}_{2}(1.54,6.31 \mathrm{mmol})$ in benzene $(\sim 45 \mathrm{ml})$ was refluxed on a fractionating column for $\sim 24 \mathrm{~h}$ during which time the liberated isopropyl alcohol was continuously fractionated out azeotropically and determined (by the oxidimetric method /18/) periodically to monitor the progress and completion of the reaction. When the azeotrope showed negligible presence of isopropyl alcohol, the reaction was stopped and the excess solvent was removed under reduced pressure to afford a yellow solid, which was recrystallized from $\mathrm{CHCl}_{3}$ and petroleum ether $\left(40-60^{\circ} \mathrm{C}\right)$ at $-20^{\circ} \mathrm{C}$ in $89 \%$ yield. Adopting a similar procedure, the rest of the compounds have also been synthesized and purified. Details of analytical data of analogous derivatives have been summarized in Table $\mathrm{I}$. Adopting a procedure similar to (2e) other derivatives were prepared from $\mathrm{Al}\left(\mathrm{OPr}^{\mathrm{i}}\right)_{3}, \mathrm{LH}_{2}$ and appropriate $\mathrm{G}(\mathrm{OH})_{2}$. The amounts of the reactants $(\mathrm{g}, \mathrm{mmol})$ used are shown in brackets:

(2a) : $\quad \mathrm{Al}\left(\mathrm{OPr}^{\prime}\right)_{3}(1.28,6.27), \mathrm{L}^{\prime} \mathrm{H}_{2}(1.44,6.27)$ and $\mathrm{G}^{1}(\mathrm{OH})_{2}(0.74,6.27)$

(2b) : $\left.\quad \mathrm{Al}_{(\mathrm{OPr}}\right)_{3}(1.44,7.05), \mathrm{L}^{2} \mathrm{H}_{2}(1.72,7.05)$ and $\mathrm{G}^{1}(\mathrm{OH})_{2}(0.83,7.05)$

(2c) : $\quad \operatorname{Al}\left(\mathrm{OPr}^{3}\right)_{3}(0.95,4.66), \mathrm{L}^{3} \mathrm{H}_{2}(1.21,4.66)$ and $\mathrm{G}^{1}(\mathrm{OH})_{2}(0.55,4.66)$

(2d): $\left.\quad \mathrm{Al}_{(\mathrm{OPr}}\right)_{3}(0.81,3.96), \mathrm{L}^{1} \mathrm{H}_{2}(0.91,3.96)$ and $\mathrm{G}^{2}(\mathrm{OH})_{2}(0.47,3.96)$

(2f) : $\left.\quad \mathrm{Al}_{(\mathrm{OPr}}\right)_{3}(1.27,6.21), \mathrm{L}^{3} \mathrm{H}_{2}(1.61,6.20)$ and $\mathrm{G}^{2}(\mathrm{OH})_{2}(0.73,6.21)$

\section{RESULTS AND DISCUSSION}

(a) Reactions of ( $\mathrm{L}) \mathrm{Al}\left(\mathrm{OPr}^{\mathrm{i}}\right)$ [which is prepared by the equimolar reactions of $\mathrm{Al}\left(\mathrm{OPr}^{\mathrm{i}}\right)_{3}$ and a variety of benzothiazoline ligands, $\mathrm{HOC}_{6} \mathrm{H}_{3} X \mathrm{C}(\mathrm{R})-\mathrm{N}(\mathrm{H}) \mathrm{C}_{6} \mathrm{H}_{4} \mathrm{~S}\left(\mathrm{LH}_{2}\right)$ \{where $\mathrm{LH}_{2}=\mathrm{L}^{\prime} \mathrm{H}_{2}(\mathrm{R}=\mathrm{X}=\mathrm{H}) ; \mathrm{L}^{2} \mathrm{H}_{2}(\mathrm{R}=$ $\left.\left.\mathrm{CH}_{3}, \mathrm{X}=\mathrm{H}\right) ; \mathrm{L}^{3} \mathrm{H}_{2}\left(\mathrm{R}=\mathrm{H}, \mathrm{X}=\mathrm{OCH}_{3}\right)\right\}$ in benzene solution] with different glycols $\mathrm{G}(\mathrm{OH})_{2}=\mathrm{G}^{\mathrm{l}}(\mathrm{OH})_{2}=$ $\mathrm{HOCMe}_{2} \mathrm{CH}_{2} \mathrm{CHMeOH}, \mathrm{G}^{2}(\mathrm{OH})_{2}=\mathrm{HOCMe}_{2} \mathrm{CMe}_{2} \mathrm{OH}$ give new mixed ligand aluminium complexes.

(i) $\mathrm{Al}\left(\mathrm{OPr}^{\mathrm{i}}\right)_{3}+\mathrm{LH}_{2}$

Benzene

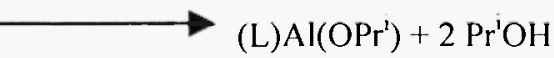

Reflux

(ii)

$$
\begin{aligned}
& \mathrm{LAl}\left(\mathrm{OPr}^{\prime}\right)+\mathrm{G}(\mathrm{OH})_{2} \longrightarrow(\mathrm{L}) \mathrm{Al}(\mathrm{OGOH})+\operatorname{Pr}^{\mathrm{i}} \mathrm{OH} \\
& \text { Reflux } \\
& \text { 1a }\left(L=L^{1}, G=G^{1}\right) ; \quad 1 b\left(L=L^{2}, G=G^{1}\right) ; \quad \text { lc }\left(L=L^{3}, G=G^{1}\right) \\
& \text { Id }\left(\mathrm{L}=\mathrm{L}^{\prime}, \mathrm{G}=\mathrm{G}^{2}\right) \text {; } 1 \mathbf{e}\left(\mathrm{L}=\mathrm{L}^{2}, \mathrm{G}=\mathrm{G}^{2}\right) ; \text { If }\left(\mathrm{L}=\mathrm{L}^{3}, \mathrm{G}=\mathrm{G}^{2}\right)
\end{aligned}
$$


(b) The compounds (OGO)Al(LH) were prepared by the reaction of (OGO)Al(OPr') and $\mathrm{LH}_{2}$ in $1: 1$ molar ratio.

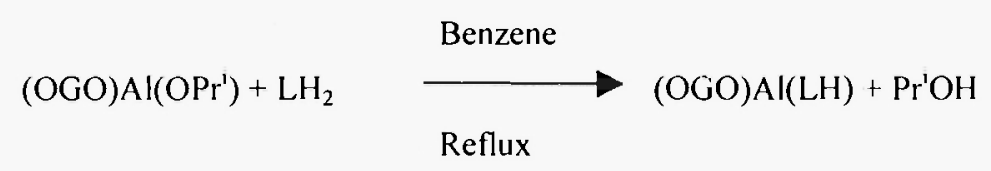

However, the above compounds may also be prepared by the equimolar reactions of $\mathrm{Al}\left(\mathrm{OPr}^{\prime}\right)_{3}, \mathrm{LH}_{2}$ and $\mathrm{G}(\mathrm{OH})_{2}$ in benzene solution.

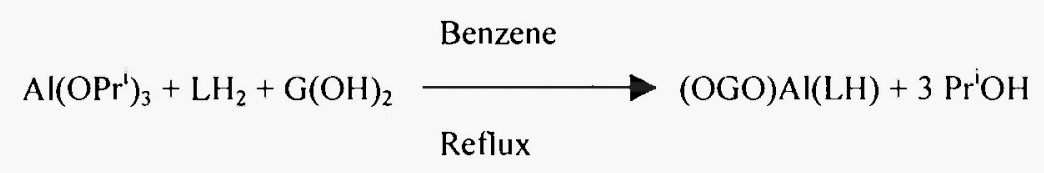

2a $\left(L=L^{1}, G=G^{1}\right) ; 2 b\left(L=L^{2}, G=G^{1}\right) ; 2 c\left(L=L^{3}, G=G^{1}\right)$

2d $\left(L=L^{1}, G=G^{2}\right) ; 2 e\left(L=L^{2}, G=G^{2}\right) ; 2 f\left(L=L^{3}, G=G^{2}\right)$

In view of the greater reactivity of phenolic $-\mathrm{OH}$ in comparision to glycolic $-\mathrm{OH}$, it is surprising that phenolic $-\mathrm{OH}$ remains unreacted in these products. It can be easily explained on the basis of the bulky $-\mathrm{NC}_{6} \mathrm{H}_{4} \mathrm{~S}$ - group in the ligands which probably decreases the reactivity of phenolic $-\mathrm{OH}$.

These two different isomers of new mixed ligand aluminium derivatives are less moisture sensitive than their parent metal alkoxide. They are yellow solids. Except lb, le, 2b, 2e, all other compounds have poor solubility in common organic solvents such as benzene and chloroform. These compounds are found to be monomeric in benzene solution.

\section{Spectroscopic Studies}

\section{Infrared Spectra}

The absence of $v_{\mathrm{S} U}$ mode $\left(\sim 2540 \mathrm{~cm}^{-1}\right)$ and the presence of $v_{\mathrm{NH}}$ mode in the range $3260-3390 \mathrm{~cm}^{-1}$ in the spectra of ligand indicates the presence of a benzothiazoline ring in the ligand. A broadening in this band is probably due to the presence of a hydrogen bond between - $\mathrm{NH}$ and phenolic $\mathrm{OH}$ group. In the spectra of complexes, the disappearance of $v_{\mathrm{NII}}$ band and the appearance of a new band at $1610-1642 \mathrm{~cm}^{-1}, 470-620$ $\mathrm{cm}^{-1}$ and 390-460 $\mathrm{cm}^{-1}$ may be assigned to $v_{\mathrm{C}=\mathrm{N}}, v_{\mathrm{Al}-\mathrm{N}} / 21 /$ and $v_{\mathrm{N}-\mathrm{S}} / 22 /$ respectively. This may be interpreted in terms of the rearrangement of the benzothiazoline ring during the formation of a Schiff base derivative of aluminium.

The spectra display a broad band at $\left(3085-3328 \mathrm{~cm}^{-1}\right)$ due to glycolic $-\mathrm{OH}$ (1 a-1 $)$ and a band of medium intensity at (3271-3457 $\left.\mathrm{cm}^{-1}\right)$ due to phenolic $-\mathrm{OH} \mathrm{(2a-2f).} \mathrm{A} \mathrm{new} \mathrm{band} \mathrm{appearing} \mathrm{at} 610-730 \mathrm{~cm}^{-1}$ has been ascribed to $v_{\mathrm{Al}-\mathrm{O}} / 21 /$ or $v_{\mathrm{Al} \leftarrow \mathrm{O}} / 23 /$ mode.

\section{'II NMR Spectra}

The 'H NMR spectra of aluminium derivatives (Table II) show the disappearance of the NH signal and a downfield shifting of $C(R)-N$ protons. This supports the rearrangement of benzothiazoline ring of the ligand during complexation. In the spectra of the compound 1a-1f, the phenolic OH proton signal is absent and a 
Table II

$\operatorname{NMR}\left({ }^{1} \mathrm{H},{ }^{27} \mathrm{Al}\right)$ data $(\delta \mathrm{ppm})$ for two different series of isomers of mixed ligand aluminium complexes.

\begin{tabular}{|c|c|c|c|c|c|c|c|c|}
\hline \multirow[t]{3}{*}{ Compound } & \multicolumn{7}{|c|}{${ }^{1} \mathbf{H}$} & \multirow[t]{3}{*}{${ }^{27} \mathbf{A i}$} \\
\hline & \multicolumn{3}{|c|}{ Schiff base moiety } & \multicolumn{4}{|c|}{ Glycolate moiety } & \\
\hline & $C R=N$ & $\begin{array}{c}\text { Aromatic } \\
\text { Proton } \\
\end{array}$ & $\begin{array}{c}\text { Phenolic } \\
\mathrm{OH}\end{array}$ & $-\mathrm{C}\left(\mathrm{CH}_{3}\right)_{2}$ & $-\mathrm{CH}\left(\mathrm{CH}_{3}\right)$ & $-\mathrm{CH}_{2}$ & $\begin{array}{c}\text { Glycolic } \\
-\mathrm{OH}\end{array}$ & \\
\hline $\begin{array}{c}\left(\mathrm{OG}^{\prime} \mathrm{OH}\right) \mathrm{Al}\left(\mathrm{L}^{1}\right) \\
(\mathbf{1} \mathbf{a}) \\
\end{array}$ & $\begin{array}{c}8.37 \\
(\mathrm{~s}, 1 \mathrm{H}) \\
\end{array}$ & $\begin{array}{c}6.53-7.98 \\
\mathrm{~m} \\
\end{array}$ & - & $\begin{array}{c}1.13 \\
(\mathrm{~s}, 6 \mathrm{H}) \\
\end{array}$ & $\begin{array}{l}4.31(\mathrm{~m}, 1 \mathrm{H}) \\
1.34(\mathrm{~d}, 3 \mathrm{H}) \\
\end{array}$ & $\begin{array}{c}1.69 \\
\text { (d, } 2 \mathrm{H}) \\
\end{array}$ & $\begin{array}{c}4.39 \\
(\mathrm{br}, 1 \mathrm{H}) \\
\end{array}$ & \\
\hline $\begin{array}{c}\left(\mathrm{OG}^{1} \mathrm{OH}\right) \mathrm{Al}\left(\mathrm{L}^{2}\right) \\
(\mathbf{1 b})\end{array}$ & $\begin{array}{r}2.37 \\
(\mathrm{~s}, 3 \mathrm{H}) \\
\end{array}$ & $\begin{array}{c}6.47-7.83 \\
\mathrm{~m}\end{array}$ & - & $\begin{array}{c}1.14 \\
(\mathrm{~s}, 6 \mathrm{H}) \\
\end{array}$ & $\begin{array}{l}4.29(\mathrm{~m}, 1 \mathrm{H}) \\
1.36(\mathrm{~d}, 3 \mathrm{H}) \\
\end{array}$ & $\begin{array}{c}1.59 \\
\text { (d. } 2 \mathrm{H}) \\
\end{array}$ & $\begin{array}{c}4.46 \\
(\mathrm{br}, 1 \mathrm{H}) \\
\end{array}$ & 25.8 \\
\hline $\begin{array}{c}\left(\mathrm{OG}^{\prime} \mathrm{OH}\right) \mathrm{Al}\left(\mathrm{L}^{3}\right)^{*} \\
(\mathbf{1 c})\end{array}$ & $\begin{array}{c}8.42 \\
(\mathrm{~s}, 1 \mathrm{H}) \\
\end{array}$ & $\begin{array}{c}6.70-7.99 \\
\mathrm{~m} \\
\end{array}$ & - & $\begin{array}{c}1.15 \\
(\mathrm{~s}, 6 \mathrm{H}) \\
\end{array}$ & $\begin{array}{l}4.01(\mathrm{~m}, 1 \mathrm{H}) \\
1.32(\mathrm{~d}, 311) \\
\end{array}$ & $\begin{array}{c}1.54 \\
\text { (d. } 2 \mathrm{H}) \\
\end{array}$ & $\begin{array}{c}4.41 \\
\text { (br, } 1 \mathrm{H}) \\
\end{array}$ & - \\
\hline $\begin{array}{c}\left(\mathrm{OG}^{2} \mathrm{OH}\right) \mathrm{Al}\left(\mathrm{L}^{1}\right) \\
(\mathbf{l d})\end{array}$ & $\begin{array}{c}8.56 \\
(s, 1 H) \\
\end{array}$ & $\begin{array}{c}\text { 6.62-7.91, } \\
\mathrm{m}\end{array}$ & - & $\begin{array}{c}1.19 \\
(\mathrm{~s}, 12 \mathrm{H}) \\
\end{array}$ & - & - & $\begin{array}{c}4.17 \\
\text { (br, 1H) }\end{array}$ & - \\
\hline $\begin{array}{c}\left(\mathrm{OG}^{2} \mathrm{OH}\right) \mathrm{Al}\left(\mathrm{L}^{2}\right) \\
(\mathbf{l e})\end{array}$ & $\begin{array}{c}2.42 \\
(\mathrm{~s}, 3 \mathrm{H})\end{array}$ & $\begin{array}{c}\text { 6.47-7.45, } \\
\mathrm{m}\end{array}$ & - & $\begin{array}{c}1.17 \\
(\mathrm{~s}, 12 \mathrm{H})\end{array}$ & - & - & $\begin{array}{c}4.31 \\
(\mathrm{br}, 1 \mathrm{H}) \\
\end{array}$ & 12.8 \\
\hline $\begin{array}{c}\left(\mathrm{OG}^{2} \mathrm{OH}\right) \mathrm{Al}\left(\mathrm{L}^{3}\right)^{*} \\
(\mathbf{1 f})\end{array}$ & $\begin{array}{c}8.10 \\
(s, 111) \\
\end{array}$ & $\begin{array}{c}6.81-7.98 \\
\mathrm{~m}\end{array}$ & - & $\begin{array}{c}1.23 \\
(\mathrm{~s}, 12 \mathrm{H}) \\
\end{array}$ & - & - & $\begin{array}{c}4.29 \\
(\mathrm{br}, 1 \mathrm{H}) \\
\end{array}$ & - \\
\hline $\begin{array}{c}\left(O G^{\prime} O\right) A l\left(L^{\prime} H\right) \\
(2 a)\end{array}$ & $\begin{array}{r}8.75 \\
(s, 111) \\
\end{array}$ & $6.4-7.99, \mathrm{~m}$ & $13.09, \mathrm{~s}$ & $\begin{array}{r}1.14 \\
(\mathrm{~s}, 6 \mathrm{H}) \\
\end{array}$ & $\begin{array}{l}4.25(\mathrm{~m}, 1 \mathrm{H}) \\
1.33(\mathrm{~d}, 3 \mathrm{H}) \\
\end{array}$ & $\begin{array}{r}1.75 \\
\text { (d. 211) } \\
\end{array}$ & - & 35.0 \\
\hline $\begin{array}{c}\left(\mathrm{OG}^{\prime} \mathrm{O}\right) \mathrm{Al}\left(\mathrm{L}^{2} \mathrm{H}\right) \\
(\mathbf{2 b})\end{array}$ & $\begin{array}{c}2.62 \\
(\mathrm{~s}, 3 \mathrm{H})\end{array}$ & $\begin{array}{c}6.4-7.98 \\
m \\
\end{array}$ & $12.55, \mathrm{~s}$ & $\begin{array}{r}1.06 \\
(\mathrm{~s}, 6 \mathrm{H})\end{array}$ & $\begin{array}{l}4.15(\mathrm{~m}, 1 \mathrm{H}) \\
1.35(\mathrm{~d}, 3 \mathrm{H})\end{array}$ & $\begin{array}{r}1.71 \\
\text { (d. } 2 \mathrm{H}) \\
\end{array}$ & - & 36.7 \\
\hline $\begin{array}{c}\left(O G^{\prime} \mathrm{O}\right) \mathrm{Al}\left(\mathrm{L}^{3} \mathrm{H}\right)^{*} \\
(2 \mathrm{c})\end{array}$ & $\begin{array}{r}8.48 \\
(s, 1[1) \\
\end{array}$ & $6.71-8.0, \mathrm{~m}$ & $13.09, \mathrm{~s}$ & $\begin{array}{r}1.07 \\
(\mathrm{~s}, 6 \mathrm{H})\end{array}$ & $\begin{array}{l}3.99(\mathrm{~m}, 1 \mathrm{H}) \\
1.32(\mathrm{~d}, 3 \mathrm{H})\end{array}$ & $\begin{array}{r}1.52 \\
\text { (d. } 2 \mathrm{H}) \\
\end{array}$ & - & - \\
\hline $\begin{array}{c}\left(\mathrm{OG}^{2} \mathrm{O}\right) \mathrm{Al}\left(\mathrm{L}^{1} \mathrm{H}\right) \\
(\mathbf{2 d})\end{array}$ & $\begin{array}{r}8.24 \\
(\mathrm{~s}, 1 \mathrm{H}) \\
\end{array}$ & $\begin{array}{c}6.78-7.99 \\
\mathrm{~m}\end{array}$ & $12.98, \mathrm{~s}$ & $\begin{array}{r}1.14 \\
(\mathrm{~s}, 12 \mathrm{H}) \\
\end{array}$ & - & - & - & $\cdot$ \\
\hline $\begin{array}{c}\left(\mathrm{OG}^{2} \mathrm{O}\right) \mathrm{Al}\left(\mathrm{L}^{2} \mathrm{H}\right) \\
(2 \mathrm{e})\end{array}$ & $\begin{array}{r}2.57 \\
(\mathrm{~s}, 3 \mathrm{H}) \\
\end{array}$ & $\begin{array}{c}6.41-7.94 \\
\mathrm{~m}\end{array}$ & $12.48, \mathrm{~s}$ & $\begin{array}{c}1.19 \\
(\mathrm{~s}, 12 \mathrm{H}) \\
\end{array}$ & - & $=$ & - & 13.0 \\
\hline $\begin{array}{c}\left(\mathrm{OG}^{2} \mathrm{O}\right) \mathrm{Al}\left(\mathrm{L}^{3} \mathrm{H}\right)^{*} \\
(2 \mathrm{f})\end{array}$ & $\begin{array}{r}8.37 \\
(\mathrm{~s}, 1 \mathrm{H}) \\
\end{array}$ & $\begin{array}{c}6.78-8.01 \\
\mathrm{~m}\end{array}$ & $13.06, \mathrm{~s}$ & $\begin{array}{c}1.20 \\
-(\mathrm{s}, 12 \mathrm{H}) \\
\end{array}$ & - & - & - & $=$ \\
\hline
\end{tabular}

* Signal for $\mathrm{OCH}_{3}$ appears at $\delta 3.99-4.08 \mathrm{ppm}$.

$s=$ singlet,$d=$ doublet, $m=$ multiplet, $b r=$ broad

glycolic $\mathrm{OH}$ proton signal is present. This indicates the deprotonation of phenolic $\mathrm{OH}$ and one of the glycolic $\mathrm{OH}$ groups, indicating the involvement of $\mathrm{OH}$ groups in the co-ordination and the formation of $\mathrm{Al} \leftarrow \mathrm{OH}$ bond. However, the spectra of compounds $2 \mathbf{a}-\mathbf{2} \mathbf{f}$ show the absence of glycolic- $\mathrm{OH}$ and the presence of phenolic $-\mathrm{OH}$. This shows that in these compounds both the glycolic protons have been deprotonated. The small shift in the position of the phenolic $-\mathrm{OH}$ group indicates the involvement of this group in the co- 
ordination and the formation of $\mathrm{Al} \leftarrow \mathrm{OH}$ bond. A multiplet in the $\delta 6.4-8.01$ region can be assigned to the aromatic protons of the Schiff base.

\section{${ }^{13}$ C NMR Spectra}

A comparison of ${ }^{13} \mathrm{C}$ NMR spectra of these aluminium derivatives (Table III) with the spectra of corresponding ligands shows deshielding of the $\mathrm{C}=\mathrm{N}$ carbon which appears at $\delta$ 162.02-169.55 ppm confirming the formation of $>\mathrm{C}=\mathrm{N} \rightarrow \mathrm{Al}$ bond by rearrangement of the benzothiazoline ring during the complexation. ${ }^{13} \mathrm{C}$ NMR spectra of all these derivatives show a downfield shift in the position of $\mathrm{C}-\mathrm{O}$ (phenolic), $\mathrm{C}-\mathrm{O}$ (glycolic) and $\mathrm{C}-\mathrm{S}$ carbon signals as compared to their respective position in the ligand moiety. However the shift in the position of $\mathrm{C}-\mathrm{O}$ (phenolic) carbon is larger in $\mathrm{Ib}$, Id and Ie as compared to $2 \mathrm{~b}$ and $2 \mathrm{e}$. This may be ascribed to the deprotonation of phenolic $-\mathrm{OH}$ in these compounds. The aromatic carbon signals of Schiff base moiety appear in the range $\delta 113.09-149.59 \mathrm{ppm}$ as multiplets.

\section{${ }^{27}$ Al NMR Spectra}

${ }^{27} \mathrm{Al}$ NMR spectra of a few representative compounds have been recorded (Table II) which show the signal in the range of $\delta 12.8-36.7 \mathrm{ppm}$. The appearance of ${ }^{27} \mathrm{Al}$ NMR signals in this region reveals a pentacoordination $/ 10,21 /$ around the central aluminium atom in the complex.

\section{Table III}

${ }^{13} \mathrm{C}$ NMR spectral data $(\delta \mathrm{ppm})$ for two series of different isomers of mixed ligand aluminium complexes.

\begin{tabular}{|c|c|c|c|c|c|c|c|c|}
\hline \multirow{2}{*}{ COMPD. } & \multicolumn{6}{|c|}{ Schiff base moiety } & \multicolumn{4}{c|}{ Glycolate moiety } \\
\cline { 2 - 9 } & $\mathbf{C H}_{3}$ & $\mathbf{C}-\mathbf{O}$ & $\mathbf{C = N}$ & Aromatic C & $-\mathbf{C H}_{3}$ & $-\mathbf{C H}_{2}$ & $-\mathbf{O C H}$ & $>$ C-O \\
\hline (1b) & 18.33 & 175.16 & 169.55 & $117.25-149.15$ & $\begin{array}{c}25.28,25.89 \\
26.21\end{array}$ & 58.31 & 65.69 & 75.94 \\
\hline (1e) & 18.32 & 175.15 & 168.98 & $115.16-148.57$ & $24.46,24.75$ & - & - & $74.75,74.93$ \\
\hline (1d) & - & 175.02 & 168.02 & $113.09-149.26$ & $24.86,25.01$ & - & - & $74.25,74.89$ \\
\hline (2b) & 18.32 & 170.02 & 162.52 & $118.25-149.28$ & $\begin{array}{c}25.82 .25 .9 \\
26.6\end{array}$ & 51.1 & 69.8 & 73.4 \\
\hline (2e) & 18.32 & 169.86 & 162.95 & $115.00-148.59$ & $24.81,25.26$ & - & - & $74.24,74.95$ \\
\hline
\end{tabular}

In view of the monomeric nature of these complexes, the presence of bifunctional tridentate ligands, glycol moiety and five-coordination around the central aluminium atom as revealed by ${ }^{27} \mathrm{Al} \mathrm{NMR,} \mathrm{the}$ following structure (Fig. I) may be proposed for the derivatives 1a-1f, in which one of the glycolic $-\mathrm{OH}$ group coordinates to central aluminium atom. 


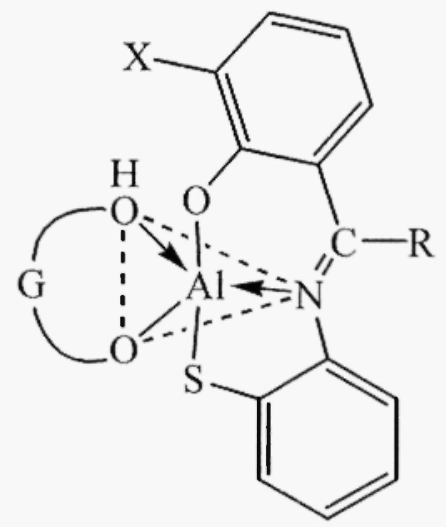

(1a-1f)

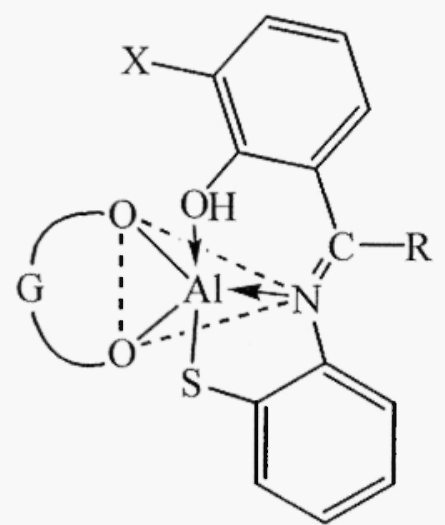

(2a-2f)

Fig. I

Fig. II

However, in view of the presence of phenolic $-\mathrm{OH}$ in the complexes $\mathbf{2 a - 2 f}$, the structure given in Fig. II, in which the central aluminium is also five co-ordinated, appears to be most plausible.

It is clear from the above evidence that the compounds of series $\mathbf{1 a - 1} \mathbf{f}$ are isomers of the compounds of series 2a-2f. This is supported by the appearance of glycolic $-\mathrm{OH}$ in the compounds $\mathbf{1 a - 1} \mathbf{f}$ and of phenolic $-\mathrm{OH}$ in the compounds $2 \mathrm{a}-2 \mathrm{f}$. The presence of these isomers has been confirmed by $\mathrm{NMR}\left({ }^{1} \mathrm{H},{ }^{13} \mathrm{C},{ }^{27} \mathrm{AI}\right)$ spectral data.

\section{ACKNOWLEDGEMENTS}

Author S.S. is grateful to the Department of Chemistry, University of Rajasthan, for a Departmental Fellowship. A.K.R. (Emeritus Fellow) and Y.P.S. are indebted to the U.G.C. for financial support.

\section{REFERENCES}

1. M.F. Garbauskas, J.H. Wengrovius, R.C. Going and J.S. Kasper, Acta Cryst., Sect C : Cryst. Struct. Commun., C40, 1536 (1984).

2. R.C. Mehrotra and A.K. Rai, Polyhedron, 10, 1967 (1991).

3. G.J. Gainsford, T. Kemmit and N.B. Milestone, Inorg. Chem., 34, 5244 (1995).

4. A. Dhammani, R. Bohra and R.C. Mehrotra, Polyhedron, 15, 733 (1996).

5. D.C. Bradley., Adv. Chem. Ser. 23, 10 (1959).

6. N. Sharma, R.K. Sharma, R. Bohra, J.E. Drake, M.B. Hursthouse and M.E. Light, J. Chem. Soc., Dalton Trans, 2002, 1631.

7. J.H. Wengrovius, M.F. Garbauskas, E.A. Williams, R.C. Going, P.E. Donahue and J.E. Smith, J. Am. Chem. Soc., 108, 982 (1986). 
8. S. Nagar, R. Bohra and R.C. Mehrotra, Synth. React. Inorg. Met. Org. Chem., 32, 1825 (2002).

9. N. Sharma, R.K. Sharma and R. Bohra, Main Group Met. Chem., 24, 781 (2001).

10. A. Dhammani, R. Bohra and R.C. Mehrotra, Polyhderon, 17, 163 (1998) and references therein.

11. A. Dhammani, R. Bohra and R.C. Mehrotra, Polyhedron, 14, 733 (1995).

12. S. Nagar, R. Bohra and R.C. Mehrotra, Main Group Met. Chem., 25, 733 (1996).

13. M. Bonamico and G. Dessy, J. Chem. Soc.A., (1968), 291.

14. U. Thewalt, F. Stollmaier, Angew. Chem., Int. Ed. Engl., 21, 133 (1982).

15. R. Benn, A. Rufinska, E.J. Lehmkuhl, E. Janssen and C. Kruger, Angew. Chem. Int. Ed. Engl., 22, 779 (1983).

16. R.C. Mehrotra, J. Indian Chem. Soc., 30, 585 (1953).

17. R.C. Mehrotra and R.K. Mehrotra, I. Indian Chem. Soc., 39, 635 (1962).

18. A.l. Vogel, A Textbook of Quantitative Inorganic Analysis, Longmans, London, 1978.

19. D.D. Perrin and W.L.F. Armarego, Purification of Laboratory Chemicals, 2nd ed., Pergamon Press, New York, 1980.

20. B.S. Saraswat, G. Srivastav and R.C. Mehrotra, J. Organomet. Chem., 137, 301 (1977).

21. S. Bansal, Y.P. Singh and A. Singh, Main. Group Met. Chem., 25, 739 (2002).

22. K.K. Chaturvedi, R.V. Singh and J.P. Tandon, Indian J. Chem., 23A, 754 (1984).

23. A. Singh, A.K. Rai and R.C. Mehrotra, Indian J. Chem., 11, 478 (1973). 
